\title{
THE FRESNEL TRIPRISM AND the circular polarization of light
}

\author{
Oriol ARTEAGA \\ Enric GARCIA-CAUREL \\ Razvigor OSSIKOVSKI \\ Departament de Física Aplicada, \\ IN2UB, Universitat de Barcelona, \\ 08028 Barcelona, Spain \\ 2 LPICM, CNRS \\ École polytechnique, \\ 91128 Palaiseau, France \\ oarteaga@ub.edu
}

\begin{abstract}
In 1822 Augustin Fresnel discovered the circular polarization of light with an experiment in which a plane polarized beam was resolved into its left- and right- circularly polarized components after refraction at slightly different angles at the interface between two different species of quartz that formed a composite prism, called the Fresnel triprism. Fresnel's landmark experiment, once popular, remains today a very little known method for producing circularly polarized light.
\end{abstract}

Indeed this was the earliest description of a Fresnel rhomb, but it would take Fresnel a few years more to realize that the light was actually circularly polarized instead of being depolarized. The concepts of linear polarization, circular polarization and elliptical polarization in the wave picture of light were established by Fresnel in the conclusion of his memoir from 1822 [4]. The main subject of this memoir was the circular double refraction of light propagating along the optic axis of a quartz crystal (cristal de roche) in an experiment that is little known today, but that was crucial for Fresnel to understand circular polarization and its relation to optical rotation. Unlike the well-known double refraction phenomenon in calcite, already well understood by Fresnel in terms of polarization, light does not split by polarization in two different paths when propagating along the optic axis of a quartz crystal. However, Fresnel thought that the phenomenon of optical rotation, first observed by Arago in

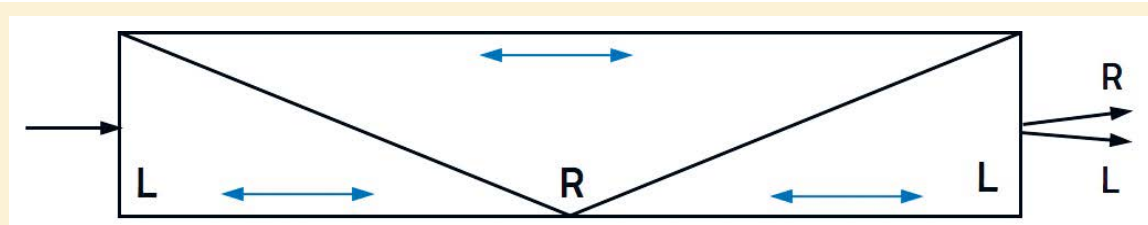

Figure 1. The Fresnel triprism. A prism made of quartz cemented in between two other half prisms made of quartz of opposite handedness splits the incident light into its right $(\mathrm{R})$ and left $(\mathrm{L})$ circularly polarized components. The double headed arrows indicate the directions of the optic axes of the crystals.
1811, could be explained by a special form of double refraction and made an experiment to demonstrate it.

Fresnel took a very acute isosceles prism of right-handed quartz and cemented it in between two half prisms of left-handed quartz, forming a composite prism, named "Fresnel triprism" and schematically shown in Figure 1. In the experiment, light always propagated substantially parallel to the optic axes of the quartz prism components, so it could not be affected by standard double refraction but, at the exit facet, Fresnel still observed two beams emerging with a small angular separation. Fresnel used double refracting calcite to check that these emerging beams were not linearly polarized as in standard double refraction, yet he checked that when the beams were totally internally reflected in a glass prism, linear polarizations were recovered, each beam being orthogonal to the other. With this experiment Fresnel postulated the circular polarization of light and understood that the optical rotation of quartz and other materials was due to the different indices of refraction for left-and right-circular polarizations. To generate the angular divergence (i.e. the circular double refraction) interfaces between quartz prisms of different species were needed, since according to Snell-Descartes's law left- and righthanded waves refract with slightly different angles at such interfaces. 


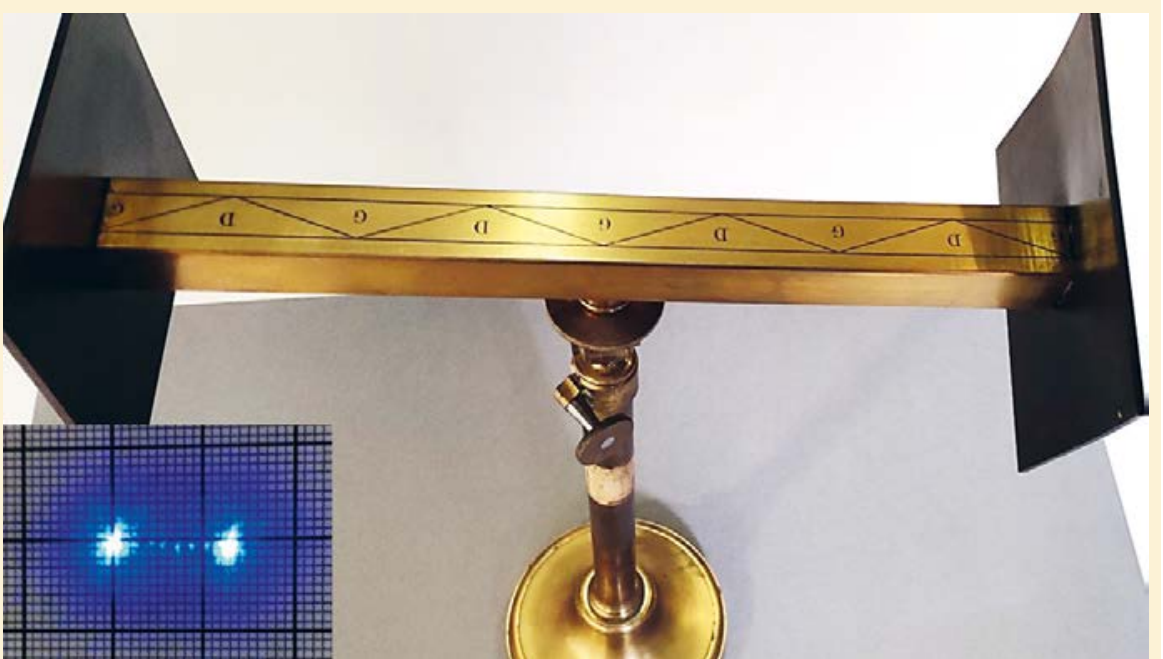

Figure 2. Photo of the historical Fresnel polyprism composed of nine prisms. The inset shows the beam splitting of a laser beam. More details in Ref. [5].
Fresnel triprisms became popular optical devices in the XIX and the first part of the XX century as theywere often used in teaching demonstrations that plane polarized light can be resolved into right-and left-circularly polarized components. However, Fresnel triprisms remain nowadays very little known optical devices and they are not available as commercial optical components. In our quest to find a Fresnel triprism we were lucky to discover that in the recently inaugurated museum of the École polytechnique (Palaiseau, France), institution where both Arago and Fresnel were trained, there is one of such composite prisms (see Fig. 2) manufactured by Henri Soleil some time before 1872 . This unit held a surprise: instead of a triprism, it contained nine different prisms of alternating handedness, so it is better referred to as a polyprism, a feature that to our knowledge is unique of this unit. Essentially, each interface doubles the angular separation between the emerging light beams, so with this device it is easy to achieve a large macroscopic separation between the right and left circularly polarized beams, something that we could test in situ in a series of experiments we performed with this historical polyprism during the summer of 2018 [5].

The Fresnel triprism, or more generally, polyprism can be regarded as the experimental culmination of what perhaps was the most fruitful decade in the history of polarization optics, started with Arago's observation of optical rotation also found in quartz. Beyond this historical significance, we think that this optical device, today almost forgotten, can be again of scientific relevance in the XXI century as methods to produce and detect circularly polarized light are becoming more and more important in today's research and technology.

\section{FURTHER READING}

[1] D.F. Arago, "Mémoire sur une modification remarquable qu'éprouvent les rayons lumineux dans leur passage à travers certains corps diaphanes, et sur quelques autres nouveaux phénomènes d'optique". Mm. Inst. vol. 1, 93-134 (1811)

[2] F. Arago and A. Fresnel, "Mémoire sur l'action que les rayons de lumière polarisée exercent les uns sur les autres". Ann. Chim. Phys. vol. 10, 288-305 (1819)

[3] A. Fresnel, "Mémoire sur les modifications que la réflexion imprime à la lumière polarisée". CEuvres vol. 1, 441-485 (1817)

[4] A. Fresnel, "Mémoire sur la double réfraction que les rayons lumineux éprouvent en traversant les aiguilles de cristal de roche suivant les directions parallèles à l'axe”. Euvres vol. 1,731-751 (1822)

[5] O. Arteaga, E. Garcia-Caurel, and R. Ossikovski, "Stern-Gerlach experiment with light: separating photons by spin with the method of A. Fresnel”. Opt. Express vol. 27, 4758-4768 (2019)

\section{Wavefront sensors and adaptive optics for optical metrology, \\ lasers and microscopy}
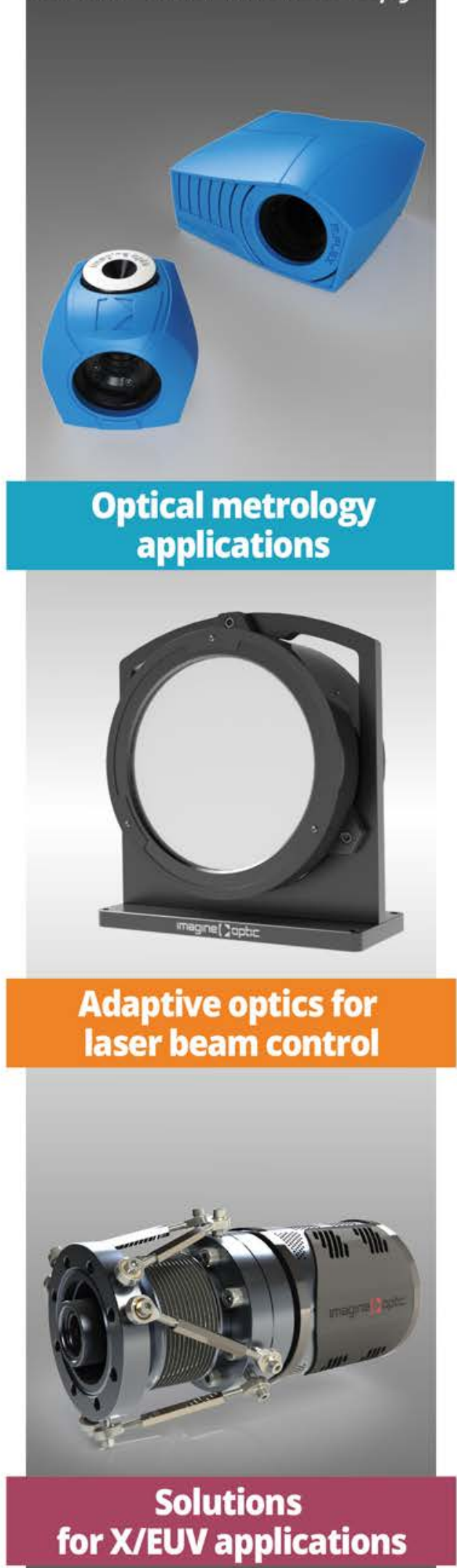

Contact us for more details: contact@imagine-optic.com or +33164861560 